\title{
Management of syphilis in times of HIV
}

\author{
"What quirk of fate has sown the seeds of this \\ dread disease, unknown for centuries, \\ but raging now through all of Europe, parts of Asia \\ and the cities of Africa?" \\ Girolamo Fracastora "Syphilis or the French Disease", \\ 1530
}

Even though effective treatment against syphilis has been available since the mid 1940s, and even though current serological diagnostic tests are highly sensitive for all but the early stages of infection, syphilis continues to be a public health problem in most countries of the world. Sexually transmitted diseases (STD) in general, and especially those characterised by genital ulceration, are epidemiologically associated with HIV infection. A number of case reports suggest that, in the presence of HIV infection, the clinical and serological natural history of syphilis may be altered, response to treatment may be less than optimal, while an increase in the incidence of neurosyphilis has been noted. ${ }^{1-4}$ The four key questions formulated by Rolfs in an excellent review article for the 1993 review of the Centers for Disease Control STD treatment recommendations remain as valid now as they were then. These are: (1) Are the manifestations of syphilis different in HIV infected individuals? (2) Is the therapy recommended for HIV negative patients effective in HIV infected individuals? (3) If manifestations or response to treatment differ, can we detect or predict which patients are at risk? (4) If current therapy is not adequate, are effective alternatives available? ${ }^{5}$ Not only are the questions still relevant, we are not much closer to answering them unequivocally. For the clinician it is also important to know whether the serological response in HIV infected individuals is different, as this would have implications for diagnosis and follow up of patients with syphilis.

Whether or not the manifestations of syphilis in HIV infected individuals are different is difficult to assess, as few comparative studies have been conducted. Although there is evidence of an increased incidence of neurosyphilis and syphilitic uveitis in HIV infected individuals, ${ }^{1}$ it is not clear what the implications of this are for management of patients. One of the few studies that has correlated complications of syphilis with the stage of HIV infection suggests that clinically apparent neurosyphilis, especially meningitis, is associated with more severe immunosuppression, ${ }^{6}$ while such an association could not be demonstrated by other authors. ${ }^{7}$ Given the importance of systemic immune responses to control disseminated infection with Treponema pallidum, it is indeed likely that immunocompromised patients are more prone to develop systemic manifestations, and will progress more rapidly to neurological complications. However, the frequency with which these complications occur in HIV infected patients is unknown, and both neurosyphilis and uveitis are also found in HIV negative individuals. And, from a patient management perspective, the most important question remains whether or not currently recommended therapy for the different stages of syphilis is effective in the presence of HIV infection.
Results of recent studies with regard to the serological and clinical response to standard treatment are equivocal, while studies cannot easily be compared, as different treatment regimens were used. While some authors report similar serological responses to treatment among HIV infected and HIV negative patients, ${ }^{8}$ others report a delayed serological response among HIV infected individuals..$^{910}$ The relevance of such a delayed serological response is not clear, however, and although there are a growing number of anecdotal reports of treatment failures in HIV infected individuals with syphilis, this finding has not been substantiated by other authors. ${ }^{9}$ However, treatment failures of syphilis in HIV negative patients occur as well, despite the use of recommended treatment regimens, although they appear to be infrequent. Moreover, it is often impossible to differentiate between a treatment failure and reinfection. From a patient management perspective, the question is whether it is possible to identify which HIV infected patients are at risk for treatment failure. These, then, could be either followed up more intensively or be given more aggressive antibiotic therapy, such as for instance treatment with intravenous aqueous crystalline penicillin $\mathrm{G}$ for a period of 10-14 days or other daily regimens with either penicillin derivatives or ceftriaxone.

It has not proved possible to identify predictors either for a slow or absent serological response to treatment or for subsequent treatment failure. ${ }^{9}$ Again, the lack of many studies to take into account the stage of HIV infection and the associated immune deficiency may be in part responsible for this, as well as the fact that treatment regimens differ between studies. Evaluation of pretreatment cerebrospinal fluid (CSF) has been proposed, but this has not been found to be predictive for treatment failure either. Besides, CSF abnormalities occur frequently in early syphilis, without evidence of neurosyphilis, and many HIV infected patients have CSF abnormalities in the absence of syphilis, making findings difficult to interpret.

What, then, are the current recommendations for management of HIV infected patients with syphilis, in the absence of predictors for potential treatment failure and in the absence of studies that allow an assessment of the frequency and magnitude of complications or the frequency of treatment failures? To these conditions should be added the knowledge that in the severely resource constrained healthcare systems in the developing world, which have to deal with the majority of such patients, alternative regimens are impractical and costly at best, and simply not feasible in most instances.

For HIV negative patients with early or early latent syphilis (of less than 1 year's duration) the recommended regimen is still $2.4 \mathrm{mU}$ benzathine penicillin $\mathrm{G}$. Treatment for patients with late latent or late syphilis, or with syphilis of unknown duration, should be with three doses of $2.4 \mathrm{mU}$ benzathine penicillin $\mathrm{G}$, at 1 week intervals. Treatment failure seems to be rare and thus does not warrant a change in the existing recommendations at this stage.

Given the lack of consistent data on either the frequency of treatment failures or of progression to neurosyphilis and other complications, treatment for HIV 
infected patients should probably be as for those who are HIV negative. However, even if the magnitude of the risk is unknown, there is a possiblity that these regimens may be insufficient to prevent progression to neurosyphilis and guarantee cure in all cases. The most practical approach, therefore, in HIV positive patients and those whose HIV serostatus is unknown is to ensure close serological and clinical follow up, with, in the absence of a satisfactory serological response, a CSF examination. This would apply equally to those patients who receive alternative, more intensive treatment regimens.

Whether or not HIV serostatus of a patient with syphilis is known, counselling and health education, including condom promotion, distribution, and demonstration should be an integral part of patient management, as should be efforts to manage sexual contacts.

Reports of serological abnormalities in the diagnosis of syphilis in HIV infected patients, such as biological false positive or false negative test results, are rare and suggest that current serological tests should continue to be used for screening and diagnosis, as well as for follow up. A slow or absent serological response should alert the clinician to the possibility of a treatment failure or reinfection. However, frequent follow up may be unrealistic in most of the developing world, and possibly even for parts of the industrialised world.

It has to be concluded that recommendations for the management of patients with syphilis, and especially for those who have concomitant HIV infection, are in need of more satisfactory and better quality evidence. This calls for carefully designed, multicentre studies, with standardised criteria for classification and staging of HIV infection and syphilis and for end points, while using standardised treatment regimens. However, in the absence of such studies, it also has to be concluded that current treatments have been and continue to be remarkably successful, and should continue to be the mainstay of clinical manage- $\Omega$ ment of syphilis.

UNAIDS/Department of Country Support, Geneva, Switzerland

1 Musher DM, Hamill RJ, Baughn RE. Effect of human immunodeficiency virus (HIV) infection on the course of syphilis and on the response to treatment. Ann Intern Med 1990;113:872-88

2 Hicks CB. Syphilis and HIV infection. Dermatol Clin 1991;9:493-501.

3 Johns DR, Tierney M, Felsenstein D. Alteration in the natural history of neurosyphilis by concurrent infection with the human immunodeficiency virus. N Engl F Med 1987;316:1569-72.

4 Katz DA, Berger JR, Duncan RC. Neurosyphilis: a comparative study of the effects of infection with human immunodeficiency virus. Arch Neuroh 1993;50:243-9.

5 Rolfs RT. Treatment of syphilis, 1993. Clin Infect Dis 1995;20(suppl 1): $\overrightarrow{0}$ S23-38.

6 Schöfer $\mathrm{H}$, Imhof $M$, Thoma-Greber E, Brockmeyer NH, Hartmann $M, \overrightarrow{-}$ Gerken G, et al. Active syphilis in HIV infection: a multicentre retrospec-W tive survey. Genitourin Med 1996;72:176-81.

7 Bordón J, Martínez-Vázquez C, Alvarez M, Miralles C, Ocampo A, de la Fuente-Aguado J, et al. Neurosyphilis in HIV-infected patients. Eur $\mathcal{F}_{\omega}$
Clin Microbiol Infect Dis 1995;14:864-9.

8 Goeman J, Kivuvu M, Nzila N, Behets F, Edidi B, Gnaore E, et al. Similarí serological response to convential therapy for syphilis among HIV-posi- $\mathrm{G}$
tive and HIV-negative women. Genitourin Med 1995;71:275-9.

9 Yinnon AM, Coury-Doniger P, Polito R, Reichman RC. Serologico response to treatment of syphilis in patients with HIV infection. Arch response to treatment of syph

10 Telzak EE, Zweig Greenberg MS, Harrison J, Stoneburner RL, Schultz S.C Syphilis treatment response in HIV-infected individuals, AIDS 1991; 5:591-5. 